\title{
Ape4
}

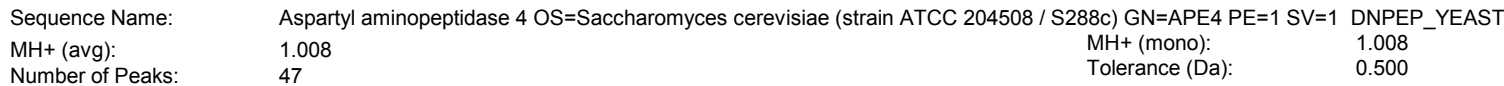

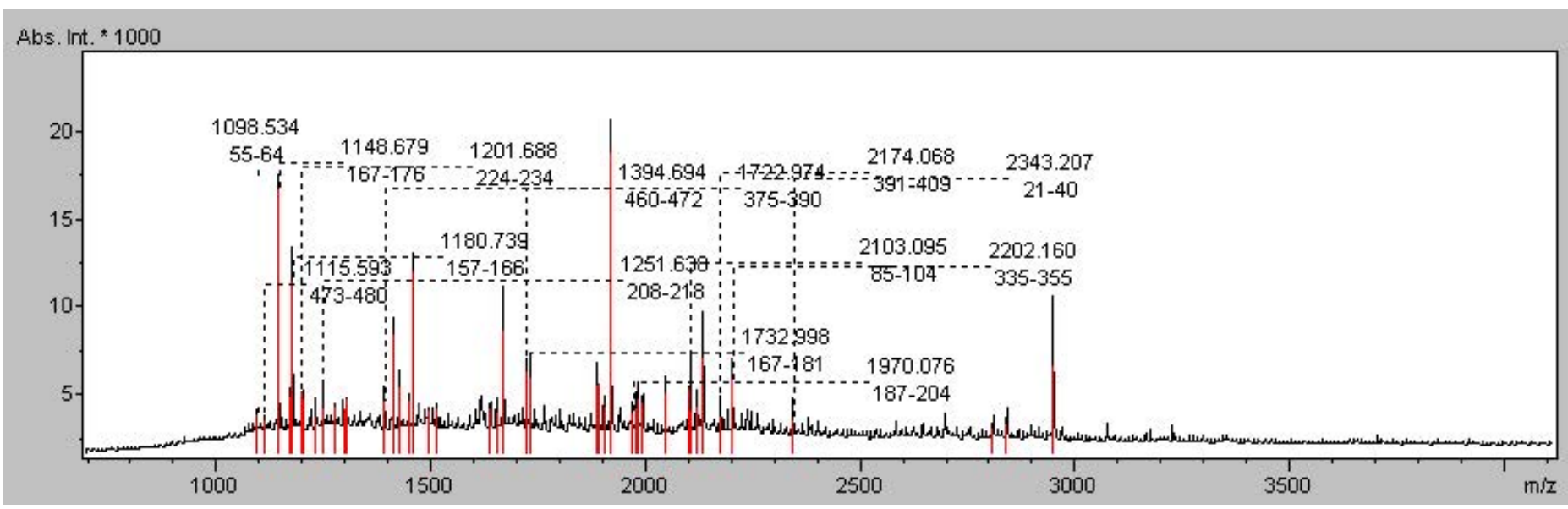

Sequence data:

\begin{tabular}{|c|c|c|c|c|c|c|c|c|c|c|}
\hline \multicolumn{2}{|c|}{ Intensity Coverage: } & \multicolumn{3}{|c|}{$31.6 \%$ (84173 cnts) } & \multicolumn{3}{|c|}{$\begin{array}{l}\text { Sequence Coverage MS: } \\
\text { pl (isoelectric point): }\end{array}$} & $\begin{array}{l}39.2 \% \\
6.6\end{array}$ & \multirow{2}{*}{\multicolumn{2}{|c|}{110}} \\
\hline 10 & 20 & 30 & 40 & 50 & 60 & 70 & 80 & 90 & & \\
\hline RIQLRTMS & SKTCKSDYPK & EFVSFLNSSH & SPYHTVHNIK & KHLVSNGF KE & LSERDSTIGH & VAQKGKYFVT & RNGSS I IAF A & VGGKWEPGNP & IAITGAHTDS & PALRIKPISK \\
\hline 120 & 130 & 140 & 10150 & 160 & 170 & 180 & 190 & 200 & 210 & 2220 \\
\hline TSEKYLQVG & VETYGGAIWH & STFDKDLGVA & GRVFVKD AKT & GKS I ARLVDL & NRPLLKIPTL & Á IHLDRDVNQ & KFEFNRETQL & LP IGGLQEDK & TEAKTE KE IN & NGEFTSIKTI \\
\hline 230 & 240 & 250 & 260 & 270 & 280 & 290 & 300 & 310 & 320 & 330 \\
\hline 2RHHAELLG & LIAKKEL A ID T & IED IEDFEL I & LYD HNASTLG & GFNDEFVFSG & RLDNLTSCFT & SMHGLTLAAD & TE IDRESGIR & LMACFDHEE I & GSSSAQGADS & NFLPN ILERL \\
\hline 340 & 350 & 360 & 370 & 380 & 390 & 400 & 410 & 420 & 430 & 440 \\
\hline LLKGDGSDQ & TKPLFHSA IL & ETSAKSFFLS & SDVAHAVHPN & YANKYESQHK & PLLGGGPVIK & INANQRYMTN & SPGLVLVKRL & ÄEALKVPLQL & FVVANDSPCG & STIGP ILASK \\
\hline 450 & 460 & 470 & 480 & 490 & 500 & & & & & \\
\hline IRTLDLGN & PVLSMHS IRE & $\begin{array}{l}\text { TGGSADLEFQ } \\
\end{array}$ & $\begin{array}{l}\text { IKLFKEFFER } \\
\end{array}$ & YTS IESE IVV & & & & & & \\
\hline
\end{tabular}

\section{Display Parameter:}

Sequence Name:

$\mathrm{MH}+(\mathrm{mono}):$

Aspartyl aminopeptidase 4 OS=Saccharomyces cerevisiae (strain ATCC 204508 / S288c) GN=APE4 PE=1 SV=1 DNPEP_YEAST

Tolerance (Da)

$\begin{array}{lcc}\text { Aspartyl aminopeptidase } & \text { OS } & \text { Saccharomyces } \\ 1.008 & \mathrm{MH}+(\text { avg): } & 1.008 \\ 0.500 & \text { Number of Peaks: } & 47\end{array}$

\section{Peaklist:}

\begin{tabular}{|c|c|c|c|c|c|c|c|c|}
\hline Peak & Mass & Intensity & Peak & Mass & Intensity & Peak & Mass & Intensity \\
\hline 1 & 1098.534 & 3898.381 & 2 & 1115.593 & 3535.283 & 3 & 1148.679 & 16578.730 \\
\hline 4 & 1174.653 & 4851.818 & 5 & 1179.587 & 5394.658 & 6 & 1180.739 & 11354.255 \\
\hline 7 & 1201.688 & 5185.014 & 8 & 1205.690 & 4837.137 & 9 & 1235.621 & 3784.635 \\
\hline 10 & 1251.638 & 4065.788 & 11 & 1252.620 & 4359.672 & 12 & 1277.707 & 4295.441 \\
\hline 13 & 1299.633 & 4242.460 & 14 & 1307.682 & 4801.136 & 15 & 1394.694 & 4652.489 \\
\hline 16 & 1414.751 & 8360.346 & 17 & 1428.764 & 5495.884 & 18 & 1451.833 & 4680.712 \\
\hline 19 & 1459.769 & 11905.383 & 20 & 1496.780 & 4128.086 & 21 & 1516.785 & 4192.999 \\
\hline 22 & 1639.918 & 4231.008 & 23 & 1654.909 & 4044.262 & 24 & 1669.910 & 8647.823 \\
\hline 25 & 1671.916 & 4573.138 & 26 & 1722.974 & 6272.862 & 27 & 1732.998 & 5947.811 \\
\hline 28 & 1888.035 & 5595.380 & 29 & 1892.024 & 5645.499 & 30 & 1904.023 & 4296.597 \\
\hline 31 & 1920.031 & 18591.713 & 32 & 1970.076 & 4005.436 & 33 & 1977.042 & 4187.863 \\
\hline 34 & 1983.125 & 4734.608 & 35 & 1994.031 & 4605.347 & 36 & 2045.943 & 5104.331 \\
\hline 37 & 2103.095 & 5425.808 & 38 & 2105.093 & 4077.560 & 39 & 2118.093 & 4336.180 \\
\hline 40 & 2133.045 & 3965.608 & 41 & 2135.109 & 7062.635 & 42 & 2174.068 & 3841.986 \\
\hline 43 & 2202.160 & 5845.170 & 44 & 2343.207 & 3564.056 & 45 & 2807.434 & 3170.576 \\
\hline 46 & 2839.414 & 3375.718 & 47 & 2947.488 & 6642.416 & & & \\
\hline
\end{tabular}

